16

\title{
Взаимодействие электромагнитной Н-волны с тонкой металлической пленкой на диэлектрической подложке в случае анизотропной поверхности Ферми металла
}

\author{
(C) И.А. Кузнецова ${ }^{1}$, Д.Н. Романов ${ }^{1, ~}$, А.А. Юшканов ${ }^{2}$ \\ ${ }^{1}$ Ярославский государственный университет им. П.Г. Демидова, \\ 150003 Ярославль, Россия \\ ${ }^{2}$ Московский государственный областной университет, \\ 105005 Москва, Россия \\ ฯ e-mail: romanov.yar357@mail.ru \\ Поступила в редакцию 27.02.2019 г. \\ В окончательной редакции 21.03.2019 г. \\ Принята к публикации 09.04.2019 г.
}

\begin{abstract}
Проведен расчет взаимодействия электромагнитной $H$-волны с тонкой металлической пленкой с учетом эллипсоидальной поверхности Ферми и постоянства длины свободного пробега электронов в случае различных углов падения электромагнитной волны $\theta$ и отличных друг от друга коэффициентов зеркальности $q_{1}$ и $q_{2}$ при отражении электронов от поверхностей пленки. Металлическая пленка заключена между двумя средами с диэлектрическими проницаемостями $\varepsilon_{1}$ и $\varepsilon_{2}$. Проведен анализ поведения коэффициентов отражения $R$, прохождения $T$ и поглощения $A$ от эффективной массы электронов проводимости.
\end{abstract}

Ключевые слова: тонкий металлический слой, коэффициенты зеркальности, диэлектрическая проницаемость, анизотропная поверхность Ферми.

DOI: $10.21883 /$ OS.2019.08.48047.78-19

\section{Введение}

Оптическим свойствам тонких проводящих пленок, в частности коэффициентам поглощения, отражения, прохождения электромагнитной волны, уделялось большое внимание [1-7]. Тонкие проводящие пленки находят широкое применение в качестве проводящих, светоотражающих, преобразующих, защитных покрытий.

Расчет оптических параметров металлической пленки проводился в работе [1] для $H$-волны и в работе [2] для $E$-волны. В данных работах пленка находится в среде с диэлектрической проницаемостью $\varepsilon=1$, поверхности пленки имеют равный коэффициент зеркальности, в электропроводности не учитывается механизм рассеяния. В работах [3] для $H$-волны и [4] для $E$-волны учитываются разные коэффициенты зеркальности поверхностей пленки и наличие разных сред, находящиеся по разные стороны от поверхностей пленки. Пространственная дисперсия и квантовые волновые свойства электронов в оптических коэффициентах учитывались с помощью зависимости диэлектрической проницаемости металла от частоты падающей электромагнитной волны в работах [5] для $H$-волны и [6] для $E$-волны. В этих работах исследуется квантовая вырожденная электронная плазма с неизменным временем релаксации в случае зеркального отражения электрона от поверхностей пленки. Оптические параметры одномерного металлодиэлектрического фотонного кристалла в случае и $E$ волны, и $H$-волны рассчитаны в [7] с учетом как пространственной дисперсии, так и квантовых волновых свойств электронной плазмы.

Настоящая работа посвящена расчету коэффициентов отражения, прохождения и поглощения электромагнитной $H$-волны тонкой металлической пленки, расположенной между двумя немагнитными диэлектрическими средами. Две поверхности пленки имеют различные коэффициенты зеркальности $q_{1}$ и $q_{2}$. Поверхность Ферми металла имеет вид трехосного эллипсоида, одна из главных осей которого перпендикулярна плоскости пленки. Эллипсоидальная изоэнергетическая поверхность является естественным обобщением более простой и часто используемой при описании явлений переноса модели сферической поверхности Ферми [8]. В данной задаче допускается, что при рассеянии электронов проводимости в объеме металла длина свободного пробега не зависит от скорости носителей заряда. Поле внутри пленки однородно, так как толщина слоя много меньше длины падающей электромагнитной волны. Толщина пленки также меньше толщины скин-слоя, поэтому скин-эффект не учитывается. На соотношение между толщиной пленки и длиной свободного пробега электронов ограничений не накладывается, квантовыми эффектами пренебрегается.

\section{Постановка задачи}

Рассмотрим тонкую металлическую пленку толщиной $a$, находящуюся между двумя различными средами. Верхняя поверхность пленки имеет коэффициент зеркальности $q_{1}$ при отражении от нее электронов 
проводимости и граничит со средой с диэлектрической поверхностью $\varepsilon_{1}$. Нижняя поверхность пленки имеет коэффициент зеркальности $q_{2}$ при отражении от нее носителей заряда и граничит с подложкой с диэлектрической поверхностью $\varepsilon_{2}$. Электромагнитная $H$-волна падает на верхнюю поверхность пленки под углом $\theta$. Среды немагнитные, величины $\varepsilon_{1}, \varepsilon_{2}$ - действительные. Уточним, что электромагнитная волна носит название „H-волна“, если вектор ее электрического поля параллелен поверхности пленки. Возьмем декартовую систему координат, начало которой находится на верхней поверхности. Электрическое поле электромагнитной $H$ волны параллельно координатной оси $Y$, координатная ось $X$ направлена вглубь тонкой металлической пленки. При таком выборе системы координат векторы напряженностей электрического и магнитного полей имеют следующую структуру:

$$
\mathbf{E}=\left\{0, E_{y}(x), 0\right\}, \quad \mathbf{H}=\left\{H_{x}(x), 0, H_{z}(x)\right\} .
$$

Далее компоненты векторов напряженностей электрического и магнитного полей ищем в виде

$$
\begin{aligned}
& E_{y}(x, z, t)=E_{y}(x) e^{-i \omega t+i k_{z} z}, \\
& H_{x}(x, z, t)=H_{x}(x) e^{-i \omega t+i k_{z} z}, \\
& H_{z}(x, z, t)=H_{z}(x) e^{-i \omega t+i k_{z} z} .
\end{aligned}
$$

Величина $k_{z}$ одинакова в двух средах и равна [4]

$$
k_{z}=\frac{\omega \sqrt{\varepsilon_{1}}}{c} \sin \theta
$$

Поведение электромагнитного поля внутри тонкого металлического слоя описывается системой уравнений [3]

$$
\left\{\begin{array}{l}
-k_{z} E_{y}=k H_{x}, \\
\frac{d E_{y}}{d x}=i k H_{z}, \\
i k_{z} H_{x}-\frac{d H_{z}}{d x}=\frac{4}{c} j_{y}-i k \varepsilon_{0} E_{y},
\end{array}\right.
$$

$k=\omega / c-$ волновое число, $c-$ скорость света, $j-$ плотность тока, $\varepsilon_{0}$ - диэлектрическая проницаемость металла.

В случае слабого внешнего электрического поля $E_{z}$ отклонение функции распределения $f$ от равновесной функции Ферми-Дирака $f_{0}$ можно представить следующим образом:

$$
\begin{aligned}
& f\left(v_{x}, x, t\right)=f_{0}(\varepsilon)+f_{1}\left(v_{x}, x, t\right) \\
&=f_{0}(\varepsilon)+f_{1}\left(v_{x}, x\right) \exp (-i \omega t), \\
& f_{1}\left(v_{x}, x\right)=-\delta\left(\varepsilon-\varepsilon_{F}\right) h\left(v_{x}, x\right),
\end{aligned}
$$

где $\varepsilon$ и $\varepsilon_{F}-$ энергия электрона проводимости и энергия ферми-поверхности соответственно.

Неравновесная функция распределения носителей заряда $f$ находится из уравнения Больцмана, линеаризованного по внешнему полю $E_{z}$, в приближении времени релаксации $\tau$ :

$$
\frac{\partial f}{\partial t}+v_{x} \frac{\partial f}{\partial x}-e E_{z} v_{z} \delta\left(\varepsilon-\varepsilon_{F}\right)=-\frac{f-f_{0}}{\tau} .
$$

Будем предполагать, что объемное время релаксации $\tau$ обратно пропорционально скорости носителя заряда (например, когда доминирует рассеяние электронов на нейтральных атомах) [9]:

$$
\tau(v)=\frac{\Lambda}{v}
$$

где $\Lambda=\mathrm{const}$ - длина свободного пробега, которая не зависит от скорости электронов проводимости.

Для кинетического уравнения будем использовать зеркально-диффузные граничные условия [4]:

$$
\left\{\begin{array}{l}
f_{1}^{+}\left(0, v_{x}\right)=q_{1} f_{1}^{-}\left(0,-v_{x}\right), \\
f_{1}^{-}\left(a,-v_{x}\right)=q_{2} f_{1}^{+}\left(a, v_{x}\right),
\end{array}\right.
$$

где $f_{1}^{+}$и $f_{1}^{-}$- отклонения от равновесной функции Ферми-Дирака для положительной и отрицательной проекций скорости на ось $X$ соответственно.

С учетом выражений (1), (2) и с учетом линеаризации слагаемого, содержащего электрическое поле, уравнение (3) упростится:

$$
\frac{\partial h}{\partial x}+\frac{e E_{z} v_{z}}{v_{x}}+\frac{w(v) h}{v_{x}}=0,
$$

где $w(v)=1 / \tau(v)-i \omega-$ величина, которую мы будем называть комплексной частотой рассеяния.

В данной работе поверхность Ферми - эллипсоид, главные оси которого совпадают с координатными осями, поэтому энергия электронов проводимости определяется следующим образом [8]:

$$
\varepsilon=\frac{m_{1} v_{x}^{2}}{2}+\frac{m_{2} v_{y}^{2}}{2}+\frac{m_{3} v_{z}^{2}}{2}
$$

где $m_{1}, m_{2}, m_{3}-$ эффективные массы квазичастицы вдоль осей $X, Y$ и $Z$ соответственно.

Проекции скорости носителей заряда в металле равны:

$$
\left\{\begin{array}{l}
v_{x}=\sqrt{\frac{2 \varepsilon}{m_{1}}} \cos \theta, \\
v_{y}=\sqrt{\frac{2 \varepsilon}{m_{2}}} \sin \theta \cos \varphi, \\
\left.v_{z}=\sqrt{\frac{2 \varepsilon}{m_{3}}} \sin \theta \sin \varphi\right),
\end{array}\right.
$$

где $\theta$ и $\varphi-$ азимутальный и полярный углы в сферической системе координат соответственно.

Для дальнейших вычислений введем следующие безразмерные параметры:

$$
V_{x}=\sqrt{\frac{m_{1}}{2 \varepsilon_{F}}} v_{x}, \quad V_{y}=\sqrt{\frac{m_{2}}{2 \varepsilon_{F}}} v_{y}, \quad V_{z}=\sqrt{\frac{m_{3}}{2 \varepsilon_{F}}} v_{z},
$$

где $V_{x}, V_{y}, V_{z}$ - безразмерные компоненты скорости.

Плотность тока определяется следующей формулой:

$$
j_{z}=e n\left\langle v_{z}\right\rangle=e \int v_{z} f \frac{2 d^{3}(m v)}{h^{3}}=2 e \frac{m_{1} m_{2} m_{3}}{h^{3}} \int v_{z} f_{1} d^{3} v,
$$

где компоненты скорости имеют вид (8), а концентрация $n$ определяется следующим выражением:

$$
n=2 \frac{m_{1} m_{2} m_{3}}{h^{3}} \int f_{0} d^{3} v=\frac{8 \pi}{3} \frac{\sqrt{m_{1} m_{2} m_{3}}}{h^{3}}\left(2 \varepsilon_{F}\right)^{3.2} .
$$




\section{Расчет высокочастотной электропроводности}

Получим выражение для электропроводности $\sigma_{z}$ тонкой металлической пленки в случае эллипсоидальной поверхности Ферми металла и постоянной длины свободного пробега электронов при рассеивании. Данное выражение нам потребуется при расчете оптических коэффициентов.

Решение уравнения (6) имеет вид

$$
h\left(x, v_{x}\right)=-\frac{e E_{z} v_{z}}{w(v)}\left(1+\psi\left(v_{x}\right) \exp \left\{-\frac{w(v) x}{v_{x}}\right\}\right)
$$

где $\psi\left(v_{x}\right)$ - постоянная интегрирования, зависящая от $v_{x}$-компоненты скорости электрона, которая находится из граничных условий (5). С учетом выражений (2) и (12) граничные условия (5) примут вид

$$
\left\{\begin{array}{l}
1+\psi^{+}\left(v_{x}\right)=q_{1}\left(1+\psi^{-}\left(-v_{x}\right)\right) \\
1+\psi^{-}\left(-v_{x}\right) \exp \left\{\frac{w(v) a}{v_{x}}\right\} \\
=q_{2}\left(1+\psi^{+}\left(v_{x}\right) \exp \left\{-\frac{w(v) a}{v_{x}}\right\}\right) .
\end{array}\right.
$$

Откуда находим

$$
\left\{\begin{array}{l}
\psi^{+}\left(v_{x}\right)=\frac{q_{1}\left(q_{2}-1\right)+\left(q_{1}-1\right) \exp \left\{\frac{w(v) a}{v_{x}}\right\}}{\exp \left\{\frac{w(v) a}{v_{x}}\right\}-q_{1} q_{2} \exp \left\{-\frac{w(v) a}{v_{x}}\right\}}, \\
\psi^{-}\left(v_{x}\right)=\frac{\left(q_{2}-1\right)+q_{2}\left(q_{1}-1\right) \exp \left\{\frac{w(v) a}{v_{x}}\right\}}{\exp \left\{-\frac{w(v) a}{v_{x}}\right\}-q_{1} q_{2} \exp \left\{\frac{w(v) a}{v_{x}}\right\}},
\end{array}\right.
$$

где $\psi^{+}$и $\psi^{-}$- функция для положительной и отрицательной проекций скорости на ось $X$ соответственно.

Подставляя выражения (2), (7)-(9), (11), (12) в (10) и переходя к сферической системе координат в пространстве скоростей, получим

$$
\begin{aligned}
j_{z} & =2 e \frac{m_{1} m_{2} m_{3}}{h^{3}} \int v_{z} f_{1} d^{3} v \\
& =\frac{3 n e^{2}}{4 \pi m_{3}} E_{z} \int_{0}^{2 \pi} \int_{0}^{\pi} \frac{\sin ^{3} \theta \sin ^{2} \varphi}{w(\theta, \varphi}\left(1+\psi\left(\sqrt{\frac{2 \varepsilon_{F}}{m_{1}}} \cos \theta\right)\right. \\
& \left.\times \exp \left\{-\frac{w(\theta, \varphi) x}{\sqrt{\frac{2 \varepsilon_{F}}{m_{1}}} \cos \theta}\right\}\right) d \theta d \varphi
\end{aligned}
$$

где

$w(\theta, \varphi)=$

$=\frac{1}{\Lambda} \sqrt{\frac{2 \varepsilon_{F}}{m_{1}} \cos ^{2} \theta+\frac{2 \varepsilon_{F}}{m_{2}} \sin ^{2} \theta \cos ^{2} \varphi+\frac{2 \varepsilon_{F}}{m_{3}} \sin ^{2} \theta \sin ^{2} \varphi}-i \omega$.

Положительная проекция скорости на ось $X$ соответствует углу $0<\theta<\pi / 2$, отрицательная $-\pi / 2<\theta<\pi$, поэтому интеграл (14) перепишется в виде

$$
\begin{aligned}
& j_{x}=\frac{3 n e^{2}}{4 \pi m_{3}} E_{z} \int_{0}^{2 \pi} \sin ^{2} \varphi d \varphi\left(\int_{0}^{\pi / 2} \frac{\sin ^{3} \theta}{w(\theta, \varphi)}\right. \\
& \times\left(1+\psi^{+}\left(\sqrt{\frac{2 \varepsilon_{F}}{m_{1}}} \cos \theta\right) \exp \left\{-\frac{w(\theta, \varphi) x}{\sqrt{\frac{2 \varepsilon_{F}}{m_{1}}} \cos \theta}\right\}\right) d \theta \\
& +\int_{\pi / 2} \frac{\sin ^{3} \theta}{w(\theta, \varphi)}\left(1+\psi^{-}\left(\sqrt{\frac{2 \varepsilon_{F}}{m_{1}}} \cos \theta\right)\right. \\
& \left.\left.\left.\times \exp \left\{-\frac{w(\theta, \varphi) x}{\sqrt{\frac{2 \varepsilon_{F}}{m_{1}}}} \cos \theta\right)\right\}\right) d \theta\right) \\
& =\frac{3 n e^{2}}{4 \pi m_{3}} E_{z} \int_{0}^{2 p i} \sin ^{2} \varphi d \varphi \int \frac{\left(1-t^{2}\right)}{w(t, \varphi)}\left(2+\psi^{+}\left(\sqrt{\frac{2 \varepsilon_{F}}{m_{1}}} t\right)\right. \\
& \left.\times \exp \left\{-\frac{w(t, \varphi) x}{\sqrt{\frac{2 \varepsilon_{F}}{m_{1}}} t}\right\}+\psi^{-}\left(-\sqrt{\frac{2 \varepsilon_{F}}{m_{1}}} t\right) \exp \left\{\frac{w(t, \varphi) x}{\sqrt{\frac{2 \varepsilon_{F}}{m_{1}}} t}\right\}\right) d t,
\end{aligned}
$$

где

$$
\begin{aligned}
& w(t, \varphi)= \\
& =\frac{\sqrt{2 \varepsilon_{F}}}{\Lambda} \sqrt{t^{2}\left(\frac{1}{m_{1}}-\frac{\cos ^{2} \varphi}{m_{2}}-\frac{\sin ^{2} \varphi}{m_{3}}\right)+\frac{\cos ^{2} \varphi}{m_{2}}+\frac{\sin ^{2} \varphi}{m_{3}}}-i \omega .
\end{aligned}
$$

и введена замена переменных $t=\cos \theta$.

В экспериментальных условиях обычно находят усредненный по толщине ток

$$
\begin{aligned}
& \left\langle j_{z}\right\rangle=\frac{1}{a} \int_{0}^{a} j_{z} d x=\frac{3 n e^{2}}{4 \pi m_{3}} E_{z} \int_{0}^{2 \pi} \sin ^{2} \varphi d \varphi \\
& \times \int_{0}^{1} \frac{\left(1-t^{2}\right)}{w(t, \varphi)}\left(2+\frac{\sqrt{\frac{2 \varepsilon_{F}}{m_{1}}} t}{a w(t, \varphi)}\right. \\
& \times \frac{\left(q_{1}+q_{2}-2\right)+\left(2 q_{1} q_{2}-q_{1}-q_{2}\right) \exp \left\{-\frac{w(t, \varphi) a}{\sqrt{\frac{2 \varepsilon_{F}}{m_{1}}} t}\right\}}{1-q_{1} q_{2} \exp \left\{-\frac{2 w(t, \varphi) a}{\sqrt{\frac{2 \varepsilon_{F}}{m_{1}}} t}\right\}} \\
& \left.\times\left(1-\exp \left\{-\frac{w(t, \varphi) a}{\sqrt{\frac{2 \varepsilon_{F}}{m_{1}}} t}\right\}\right)\right) d t .
\end{aligned}
$$

Ток $\langle j z\rangle$ связан с напряженностью $E_{z}$ через удельную проводимость $\sigma_{z}$ из локального закона Ома:

$$
\left\langle j_{z}\right\rangle=\sigma_{z} E_{z}
$$


Введем безразмерные переменные

$$
\begin{gathered}
\sigma_{0}=\frac{n e^{2} \Lambda}{m_{0} v_{0}}, \quad m_{0}=\sqrt{3} m 1 m_{2} m_{3}, \quad v_{0}=\sqrt{\frac{2 \varepsilon_{F}}{m_{0}}}, \\
k_{m 1}=\frac{m_{1}}{m_{0}}, \quad k_{m 2}=\frac{m_{2}}{m_{0}}, \quad k_{m 3}=\frac{m_{3}}{m_{0}}, \\
z_{0}=\frac{w a}{v_{0}}==x_{0} \\
\times \sqrt{t^{2}\left(k_{m 2} k_{m 3}-\frac{\cos ^{2} \varphi}{k_{m 2}}-\frac{\sin ^{2} \varphi}{k_{m 3}}\right)+\frac{\cos ^{2} \varphi}{k_{m 2}}+\frac{\sin ^{2} \varphi}{k_{m 3}}}-i y_{0}, \\
x_{0}=\frac{a}{\Lambda}, \quad y_{0}=\frac{a}{v_{0}} .
\end{gathered}
$$

Здесь $k_{m 1}, k_{m 2}, k_{m 3}$ - безразмерные эффективные массы вдоль осей $X, Y$ и $Z$ соответственно; $v_{0}$ - эффективная скорость; $x_{0}$ - безразмерная толщина пленки; $y_{0}-$ безразмерная частота электрического поля.

Отдельно стоит отметить взаимосвязь безразмерных эффективных масс; так как $m_{0}=\sqrt{3} m_{1} m_{2} m_{3}$, то $k_{m 1} k_{m 2} k_{m 3}=1$, поэтому $k_{m 1}=1 /\left(k_{m 2} k_{m 3}\right)$. Также при изменении безразмерных эффективных масс предполагается постоянство концентрации свободных носителей заряда (11), следовательно, $m 1 m 2 m 3=$ const, $m 0=$ const, a $k_{m 1} \propto m 1, k_{m 2} \propto m 2, k_{m 3} \propto m 3$.

Выражение для электропроводности после подстановки безразмерных параметров и с учетом (15) и (16) выглядит следующим образом:

$$
\begin{aligned}
& \sigma_{z}=\frac{3 x_{0}}{4 \pi k_{m} 3} \sigma_{0} \int_{0}^{2 \pi} \sin ^{2} \varphi d \varphi \int_{0}^{1} \frac{\left(1-t^{2}\right)}{z_{0}}\left(2-\frac{t}{z_{0}} \sqrt{k_{m 2} k_{m 3}}\right. \\
& \times \frac{2-\left(q_{1}+q_{2}\right)+\left(q_{1}+q_{2}\right) \exp \left\{-\frac{z_{0}}{t \sqrt{k_{m 2} k_{m 3}}}\right\}-2 q_{1} q_{2} \exp \left\{-\frac{z_{0}}{t \sqrt{k_{m 2} k_{m 3}}}\right\}}{1-q_{1} q_{2} \exp \left\{-\frac{2 z_{0}}{t \sqrt{k_{m 2} k_{m 3}}}\right\}} \\
& \left.\times\left(1-\exp \left\{-\frac{z_{0}}{t \sqrt{k_{m 2} k_{m 3}}}\right\}\right)\right) d t .
\end{aligned}
$$

\section{Коэффициенты отражения $R$, поглощения $A$ и прохождения $T$}

Коэффициенты прохождения $T$, отражения $R$ и поглощения $A$ электромагнитной волны слоем описываются следующими выражениями [3]:

$$
\begin{gathered}
T=\frac{1}{4}\left|P^{(1)}-P^{(2)}\right|^{2}, \\
R=\frac{1}{4}\left|P^{(1)}+P^{(2)}\right|^{2}, \\
A=1-T-R .
\end{gathered}
$$

Величины $P^{(1)}$ и $P^{(2)}$ связаны с поверхностным импедансом металлической пленки $Z^{(1)}$ и $Z^{(2)}$, с углом падения электромагнитной $H$-волны $\theta$ и со значением диэлектрической проницаемости среды $\varepsilon_{1}$ [3]:

$$
\begin{aligned}
& P^{(1)}=\frac{\sqrt{\varepsilon_{1}} Z^{(1)} \cos \theta-1}{\sqrt{\varepsilon_{1}} Z^{(1)} \cos \theta+1}, \\
& P^{(2)}=\frac{\sqrt{\varepsilon_{1}} Z^{(2)} \cos \theta-1}{\sqrt{\varepsilon_{1}} Z^{(2)} \cos \theta+1},
\end{aligned}
$$

$Z^{(1)}$ и $Z^{(2)}$ соответствуют импедансу на нижней поверхности тонкой металлической пленки. $Z^{(1)}$ соответствует антисимметричной по электрическому полю конфигурации внешнего поля: $E_{y}(0)=-E_{y}(a), H_{z}(0)=H_{z}(a)$, а $Z^{(2)}$ - симметричной по внешнему полю конфигурации внешнего поля: $E_{y}(0)=E_{y}(a), H_{z}(0)=-H_{z}(a)$.

Выражения для поверхностного импеданса в случае взаимодействия $H$-волны с тонкой металлической пленкой были получены в работе [1] в предположении, что длина волны падающего излучения существенно превосходит толщину слоя,

$$
\begin{gathered}
Z^{(1)}=0, \\
Z^{(2)}=\frac{c}{2 \pi a \sigma_{z}} .
\end{gathered}
$$

Окончательные выражения для коэффициента отражения $R$, коэффициента прохождения $T$ и коэффициента поглощения $A$ были получены в [3], учитывая выражения (17)-(21):

$$
\begin{gathered}
R=\left|\frac{\sqrt{\varepsilon_{1,2}-\sin ^{2} \theta}\left(\bar{P}+P^{(1)} P^{(2)}\right)+\cos \theta\left(\bar{P}-P^{(1)} P^{(2)}\right.}{\sqrt{\varepsilon_{1,2}-\sin ^{2} \theta}(1+\bar{P})+\cos \theta(1-\bar{P})}\right|^{2}, \\
T=\cos \theta \operatorname{Re} \sqrt{\varepsilon_{1,2}-\sin ^{2} \theta} \\
\times\left|\frac{P^{(2)}-P^{(1)}}{\sqrt{\varepsilon_{1,2}-\sin ^{2} \theta}(1+\bar{P})+\cos \theta(1-\bar{P})}\right|^{2} \\
A=1-T-R, \\
\varepsilon_{1,2}=\frac{\varepsilon_{2}}{\varepsilon_{1}}, \quad \bar{P}=\frac{1}{2}\left(P^{(1)}+P^{(2)}\right) .
\end{gathered}
$$

\section{Анализ результатов}

Рассмотрим особенности поведения коэффициентов отражения $R$, поглощения $A$ и прохождения $T$ в зависимости от безразмерной эффективной массы носителей заряда. Во всех случаях $\varepsilon_{1}=1$, так как электромагнитная волна падает на тонкую металлическую пленку из вакуума, $\varepsilon_{2}=5$ (стекло). Пусть $\varepsilon_{F}=5 \mathrm{eV}, \Lambda=50 \mathrm{~nm}$, $n=10^{28} \mathrm{~cm}^{-3}$ (типичные значения для металла). Толщина пленки равна $a=10 \mathrm{~nm}$. Электромагнитная волна падает на пленку под углом $\theta=20^{\circ}$. Частота электромагнитной волны лежит в дальнем инфракрасном диапазоне: $f=1 \mathrm{THz}$. 


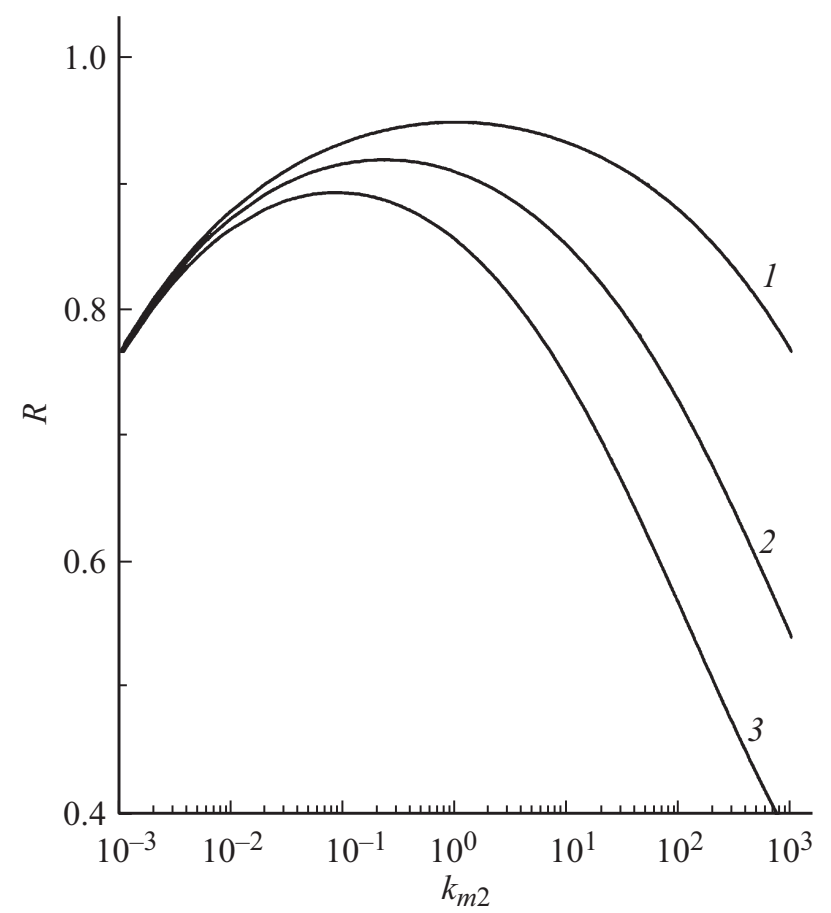

Pис. 1. Зависимость коэффициента отражения $R(22)$ от параметра $k_{m 2}$ при $k_{m 3}=1$. Кривые $1-q_{1}=q_{2}=1 ; 2-$ $q_{1}=0.5, q_{2}=0.6 ; 3-q_{1}=q_{2}=0$.

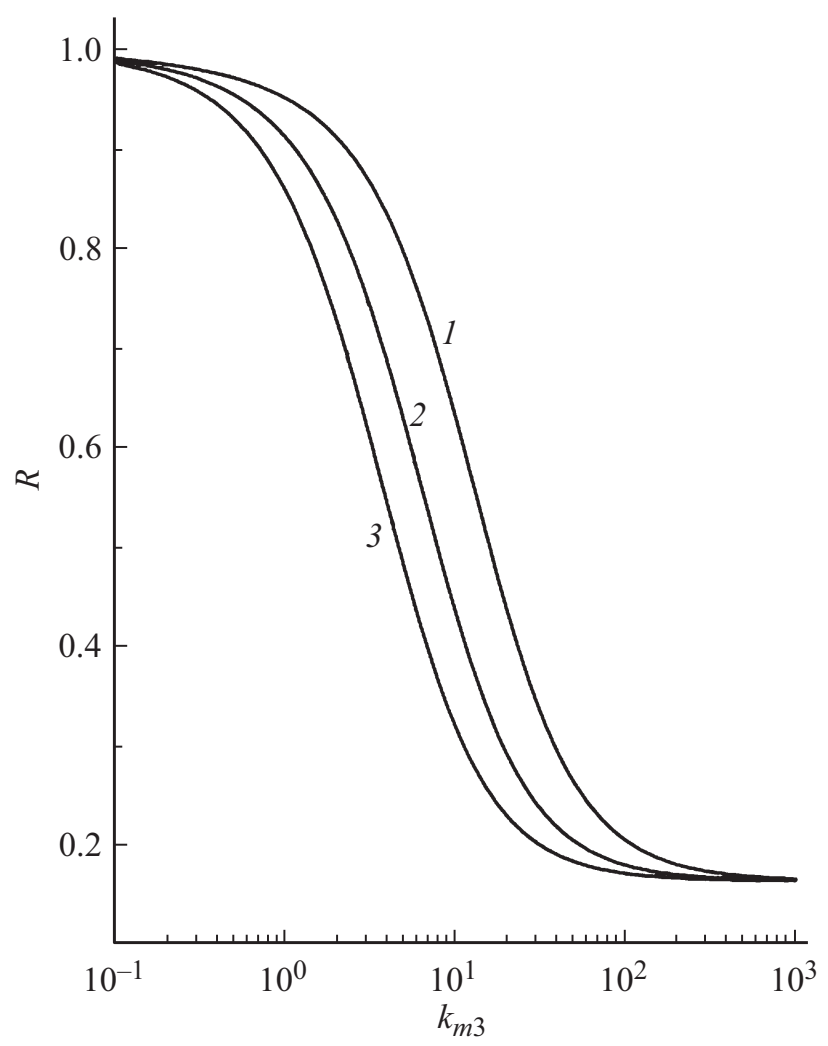

Pис. 2. Зависимость коэффициента отражения $R(22)$ от параметра $k_{m 3}$ при $k_{m 2}=1$. Кривые $1-q_{1}=q_{2}=1 ; 2-$ $q_{1}=0.5, q_{2}=0.6 ; 3-q_{1}=q_{2}=0$.

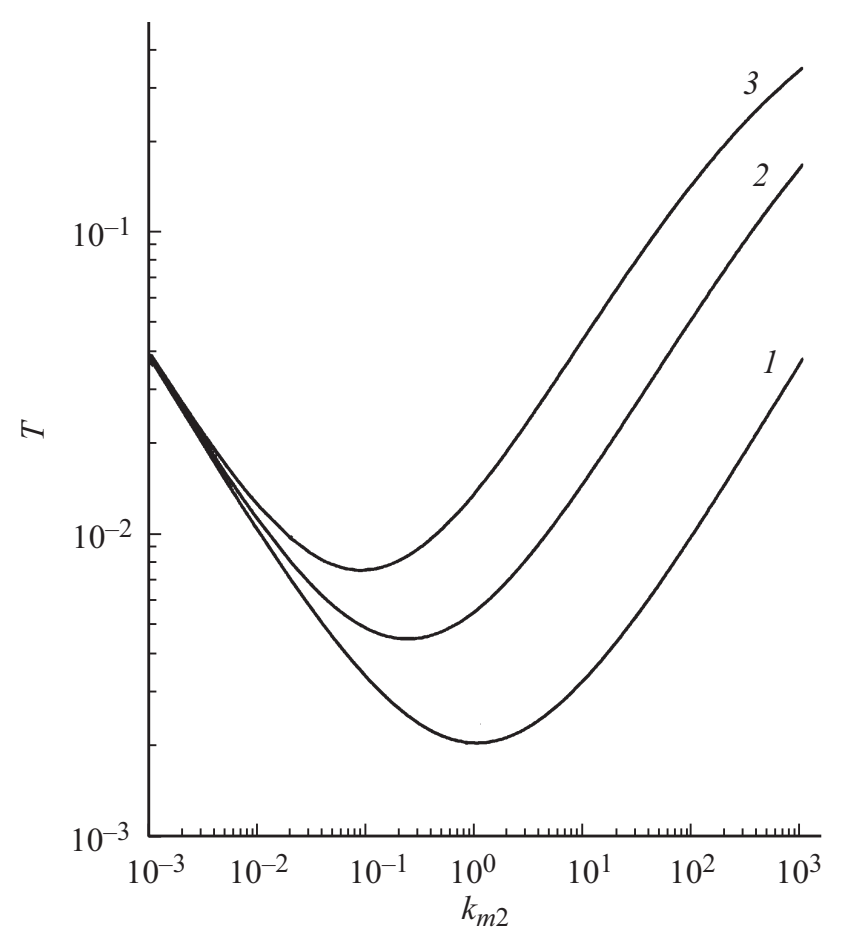

Рис. 3. Зависимость коэффициента прохождения $T$ (23) от параметра $k_{m 2}$ при $k_{m 3}=1$. Кривые $1-q_{1}=q_{2}=1 ; 2-$ $q_{1}=0.5, q_{2}=0.6 ; 3-q_{1}=q_{2}=0$.

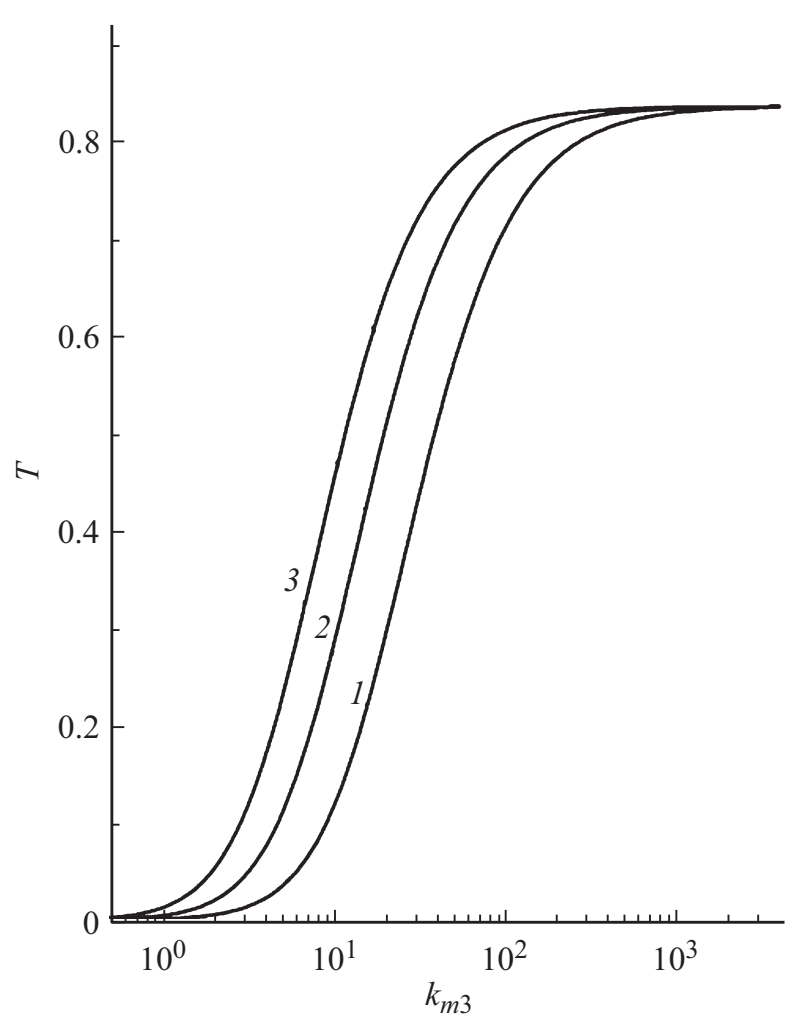

Рис. 4. Зависимость коэффициента прохождения $T$ (23) от параметра $k_{m 3}$ при $k_{m 2}=1$. Кривые $1-q_{1}=q_{2}=1 ; 2-$ $q_{1}=0.5, q_{2}=0.6 ; 3-q_{1}=q_{2}=0$. 


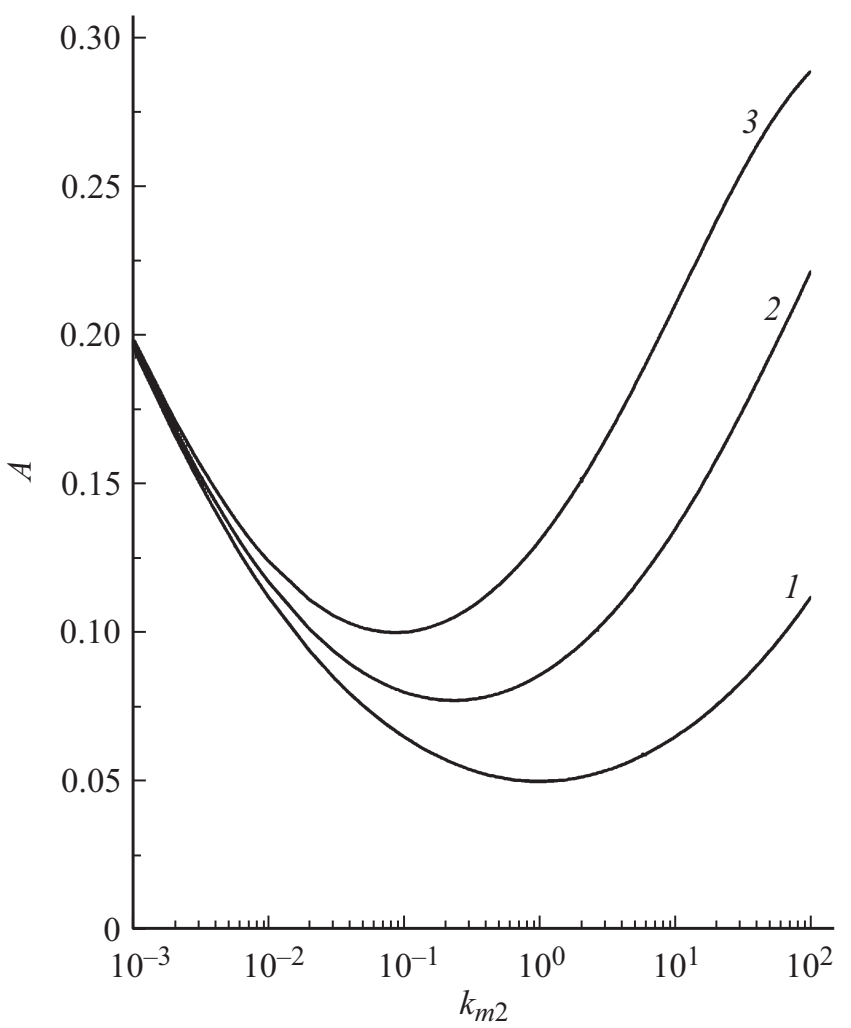

Рис. 5. Зависимость коэффициента поглощения $A$ (24) от параметра $k_{m 2}$ при $k_{m 3}=1$. Кривые $1-q_{1}=q_{2}=1 ; 2-$ $q_{1}=0.5, q_{2}=0.6 ; 3-q_{1}=q_{2}=0$.

На рис. 1 изображена зависимость коэффициента отражения $R(22)$ от безразмерной эффективной массы $k_{m 2}$ вдоль оси $Y$ при различных коэффициентах зеркальности $q_{1}$ и $q_{2}$. У данной зависимости наблюдается максимум, который смещается в сторону более высоких значений $k_{m 2}$ при увеличении коэффициентов зеркальности. Если $k_{m 2} \rightarrow 0$, то величина электропроводности, следовательно, и оптические коэффициенты не зависят от коэффициентов зеркальности.

На рис. 2 представлена зависимость коэффициента отражения $R(22)$ от безразмерной эффективной массы $k_{m 3}$ вдоль оси $Z$ при различных коэффициентах зеркальности $q_{1}$ и $q_{2}$. С ростом эффективной массы вдоль оси $Y$ или с уменьшением коэффициентов зеркальности $q_{1}$ и $q_{2}$ электропроводность и, как следствие, коэффициент отражения снижаются. В случае $k_{m 3} \rightarrow 0$ металл идеально отражает $(R \rightarrow 1)$.

На рис. 3 показана зависимость коэффициента прохождения $T$ (23) от безразмерной эффективной массы $k_{m 2}$ вдоль оси $Y$ при различных коэффициентах зеркальности $q_{1}$ и $q_{2}$. Эти кривые имеют минимум, который сдвигается в сторону более высоких значений $k_{m 2}$ при росте коэффициентов зеркальности.

На рис. 4 видно, что коэффициент прохождения $T$ (23) при различных коэффициентах зеркальности $q_{1}$ и $q_{2}$ возрастает с увеличением безразмерной эффективной массы $k_{m 3}$ вдоль оси $Z$. Рост коэффициента прохождения

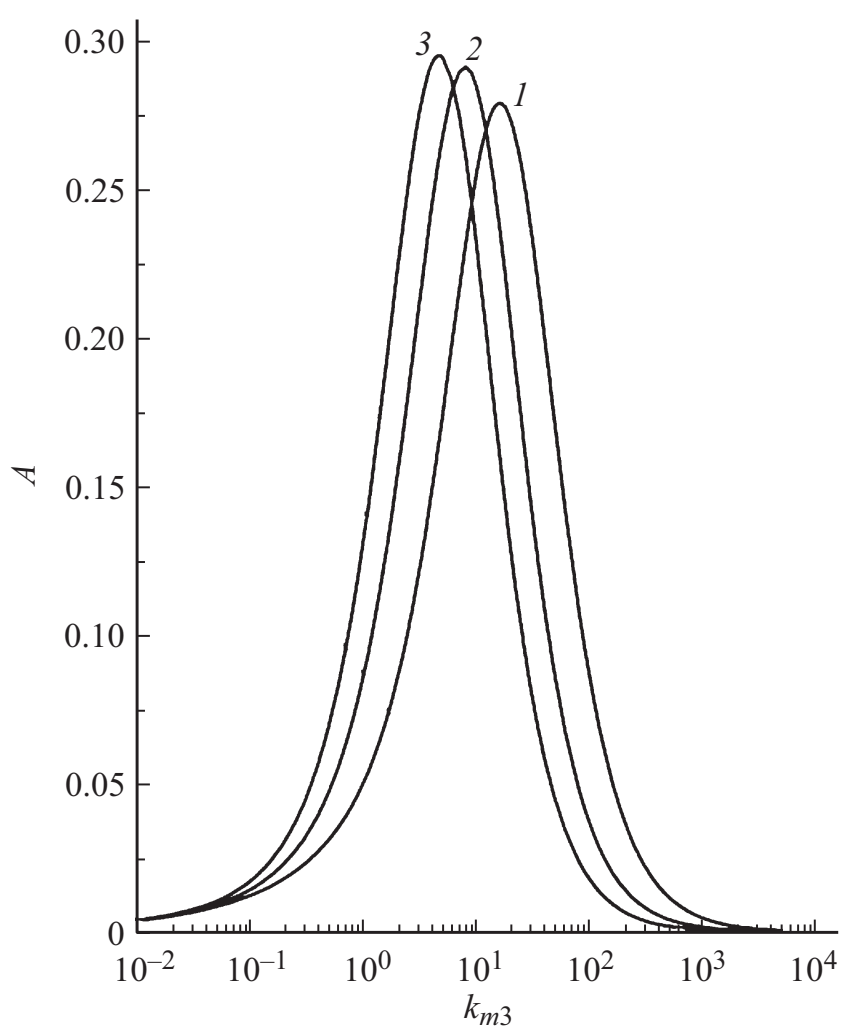

Рис. 6. Зависимость коэффициента поглощения $A$ (24) от параметра $k_{m 3}$ при $k_{m 2}=1$. Кривые $1-q_{1}=q_{2}=1 ; 2-$ $q_{1}=0.5, q_{2}=0.6 ; 3-q_{1}=q_{2}=0$.

с увеличением эффективной массы вдоль оси $Z$ или с уменьшением коэффициентов зеркальности обусловлен низкой скоростью носителей заряда, которые не успевают экранировать внешнюю электромагнитную волну.

На рис. 5 изображена зависимость коэффициента поглощения $A$ (24) от безразмерной эффективной массы $k_{m 2}$ вдоль оси $Y$ при различных коэффициентах зеркальности $q_{1}$ и $q_{2}$. На данном графике наблюдается минимум кривых, который смещается в сторону больших значений $k_{m 2}$ при росте коэффициентов зеркальности.

На рис. 6 представлена зависимость коэффициента поглощения $A(24)$ от безразмерной эффективной массы $k_{m 3}$ вдоль оси $Z$ при различных коэффициентах зеркальности $q_{1}$ и $q_{2}$. Даная зависимость имеет максимум, который при увеличении $q_{1}$ и $q_{2}$ снижается по величине и смещается в сторону более высоких значений $k_{m 3}$. Коэффициент поглощения стремится к нулю при $k_{m 3} \rightarrow 0$ и $k_{m 3} \rightarrow \infty$.

\section{Заключение}

Таким образом, в настоящей работе показано влияние эффективной массы электронов проводимости в тонкой металлической пленке на оптические коэффициенты при взаимодействии $H$-волны с этой пленкой. У зависимости коэффициента отражения от эффективной массы 
вдоль оси $Y$ наблюдается максимум, а у коэффициентов прохождения и поглощения - минимум. Эти точки экстремума оптических коэффициентов смещаются в сторону больших значений эффективной массы вдоль оси $Z$ при росте коэффициентов зеркальности поверхностей пленки. При малой эффективной массе вдоль оси $Z$ электропроводность, следовательно, и оптические коэффициенты не зависят от коэффициентов зеркальности. С ростом эффективной массы вдоль оси $Y$ или с уменьшением коэффициентов зеркальности $q_{1}$ и $q_{2}$ электропроводность уменьшается и, как следствие, коэффициент отражения снижается, а коэффициент прохождения возрастает. Коэффициент поглощения от эффективной массы вдоль оси $Z$ имеет максимум, который при увеличении коэффициентов зеркальности уменьшается по величине и смещается в сторону более высоких значений эффективной массы вдоль оси $Z$.

\section{Конфликт интересов}

Авторы заявляют, что у них нет конфликта интересов.

\section{Список литературы}

[1] Латышев А.В., Юшканов А.А. // Микроэлектроника. 2012. T. 41. № 1. C. 30.

[2] Латышев А.В. // Опт. и спектр. 2011. Т. 110. № 5. С. 796.

[3] Utkin A.I., Yushkanov A.A. // J. Phys. Chem. Sol. 2015. V. 16. N 2. P. 253. doi 10. $15330 /$ pcss.16.2.253-256

[4] Уткин А.И., Юшканов А.А. // Опт. и спектр. 2018. Т. 124. № 2. C. 250. doi 10.21883/0000000000

[5] Yushkanov A.A., Zverev N.V. // arXiv: 1709.02240v1 [physics.plasm-ph] 5 Sep 2017.

[6] Yushkanov A.A., Zverev N.V. // Phys. Lett. 2017. V. 281. P. 679.

[7] Зверев Н.В., Юшканов А.А. // Опт. и спектр. 2017. Т. 122. № 2. C. 222. doi 10.7868/S0030403417020271

[8] Абрикосов А.А. Основы теории металлов. М.: Наука, 1987. $520 \mathrm{c}$.

[9] Латышев А.В., Юиканов А.А. // Физика плазмы. 2006. T. 32. № 11. C. 1021. 